\title{
Fluid substitution in a shaley sandstone reservoir at seismic scale
}

\author{
Li Jingye ${ }^{1,2 *}$
}

\author{
${ }^{1}$ State Key Laboratory of Petroleum Resource and Prospecting, China University of Petroleum, Beijing 102249, China \\ ${ }^{2}$ CNPC Key Laboratory of Geophysical Exploration, China University of Petroleum, Beijing 102249, China \\ (C) China University of Petroleum (Beijing) and Springer-Verlag Berlin Heidelberg 2012
}

\begin{abstract}
Shaley sandstone is heterogeneous at a seismic scale. Gassmann's equation is suited for fluid substitution in a homogeneous medium. To study the difference between shaley sandstone effective elastic moduli calculated by mean porosity as a homogeneous medium, and those calculated directly from the sub-volumes of the volume as a heterogeneous medium, computational experiments are conducted on Han's shaley sand model, the soft-sand model, the stiff-sand model, and their combination under the assumption that the shaley sandstone volume is made up of separate homogenous sub-volumes with independent porosity and clay content. Fluid substitutions are conducted by Gassmann's equation on rock volume and sub-volumes respectively. The computational data show that at seismic scale, there are minor differences between fluid substitution on rock volume and that on sub-volumes using Gassmann's equation. But fluid substitution on sub-volumes can take consideration of the effects of low porosity and low permeability sub-volumes, which can get more reasonable data, especially for low porosity reservoirs.
\end{abstract}

Key words: Fluid substitution, Gassmann's equation, shaley sandstone, seismic scale

\section{Introduction}

Gassmann's equation is widely used to predict elastic moduli of a porous rock saturated with a given fluid based on the corresponding properties of the dry rock, which is called fluid substitution. Gassmann's equation makes the following assumptions: (1) a homogenous mineral modulus and statistical isotropy of the pore space; (2) seismic lowfrequency so the pore pressures are equilibrated throughout the pore space; (3) all minerals making up the rock have the same bulk and shear moduli; (4) fluid bearing rock is completely saturated (Gassmann, 1951). For the low frequency assumption, the seismic frequency is generally acceptable. But at seismic scale, the shaley sandstone is rarely homogenous (Skelt, 2004; Dvorkin and Uden, 2006; Dvorkin et al, 2007), because many factors can cause rock heterogeneity, including depositional environment variation and the difference in compaction, diagenesis and cementation (Blangy, 1992). We often apply mean porosity in fluid substitution by Gassmann's equation to obtain the effective bulk and shear moduli saturated with different fluids. But what is the difference between effective elastic moduli calculated by mean porosity, and those calculated directly from the sub-volumes of rock. Since the shear moduli are constant for a rock saturated with different fluids, in the paper,

*Corresponding author. email: lijingye@cup.edu.cn Received April 6, 2011 we mainly study differences between bulk moduli calculated by mean porosity, and those calculated directly from the subvolumes of rock.

To study the question, we conduct computational experiments, on the supposition that (1) the shaley sandstone volume is made up of separate homogenous sub-volumes; (2) porosity $(\phi)$ and clay content $(C)$ for each sub-volume of the whole rock volume are independent and follow a Gaussian probability distribution function, shown as Fig. 1. First, we get both porosity and clay content by Monte Carlo simulation (Avseth et al, 2005). Next, we use rock physical models, including Han's shaley sandstone model (Han, 1987), the soft-sand model, the stiff-sand model (Mavko et al, 2009) and their combination, to compute the dry rock moduli of each sub-volume. Then we compute the bulk moduli of water-saturated rock by two ways. In the first way, on the supposition that the shaley sandstone volume is homogenous, we compute the volume moduli of water-saturated rock using mean porosity by Gassmann's equation, shown as Eq. (1). During the calculation, the volume moduli of dry rock are obtained from the sub-volumes by generalized HashinShtrikman-Walpole bounds (Berryman, 1995). In the second way, on the supposition that each component of the shale sandstone volume is homogenous, we compute each subvolume modulus of water-saturated rock by Gassmann's equation from the dry moduli of the sub-volumes, and obtain the effective bulk moduli by generalized Hashin-ShtrikmanWalpole bounds (Berryman, 1995). Then we analyze and compare the data computed in two different ways. 


$$
K_{\text {Wet }}=K_{\mathrm{s}} \frac{\varphi K_{\mathrm{Dry}}-(1+\varphi) K_{\mathrm{f}} K_{\mathrm{Dry}} / K_{\mathrm{s}}+K_{\mathrm{f}}}{(1-\varphi) K_{\mathrm{f}}+\varphi K_{\mathrm{s}}-K_{\mathrm{f}} K_{\text {Dry }} / K_{\mathrm{s}}}
$$

In above equation, $\varphi$ is porosity, and $K_{\text {Wet }}, K_{\mathrm{s}}, K_{\mathrm{Dry}}, K_{\mathrm{f}}$, are the bulk moduli of saturated rock, mineral grain, rock framework and fluid. If we specify only the moduli of the rock constituent and its volume fractions, we can predict upper and lower shear and bulk moduli bounds by the generalized form of Hashin-Shtrikman-Walpole bounds (Berryman, 1995), shown as Eq. (2). We use Eq. (2) to compute the bulk moduli bounds during our computational experiments.

$$
\begin{aligned}
& K^{\mathrm{HS}+}=\Lambda\left(G_{\max }\right) \\
& K^{\mathrm{HS}-}=\Lambda\left(G_{\text {min }}\right) \\
& G^{\mathrm{HS}+}=\Gamma\left(\zeta\left(K_{\max }, G_{\text {max }}\right)\right) \\
& G^{\mathrm{HS}-}=\Gamma\left(\zeta\left(K_{\text {min }}, G_{\min }\right)\right) \\
& \Lambda(z)=\left\langle\frac{1}{K(r)+\frac{4}{3} z}\right\rangle^{-1}-\frac{4}{3} z \\
& \Gamma(z)=\left\langle\frac{1}{G(r)+z}\right\rangle^{-1}-z \\
& \zeta(K, G)=\frac{G}{6}\left(\frac{9 K+8 G}{K+2 G}\right)
\end{aligned}
$$

In above equation, $K$ and $G$ are the bulk and shear moduli of sub-volumes. $K^{\mathrm{HS}+}$ and $G^{\mathrm{HS}+}$ are the upper bounds of bulk and shear moduli, and $K^{\mathrm{HS}-}$ and $G^{\mathrm{HS}-}$ are the lower bounds of bulk and shear moduli. \langle\rangle is taking the average.

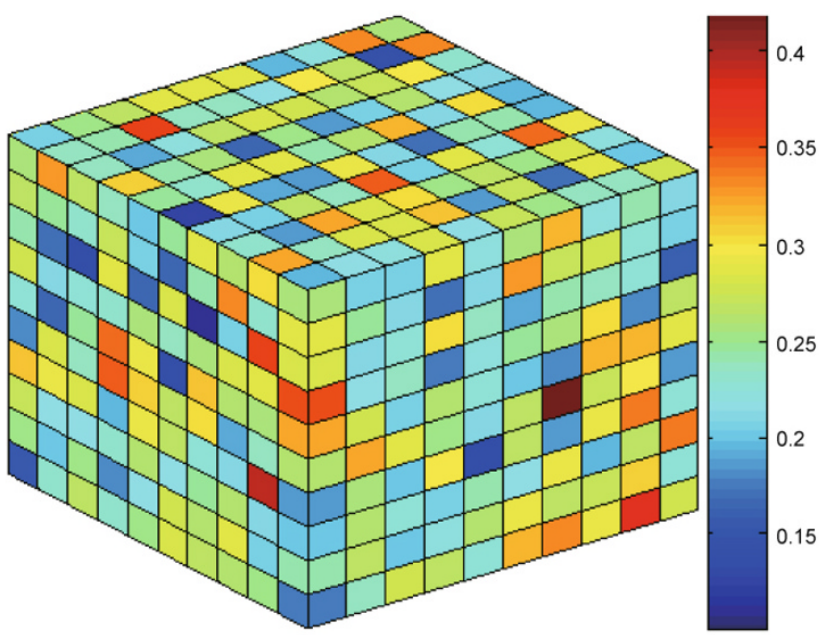

Fig. 1 The color-coded porosity of separate homogenous sub-volumes of the whole rock volume. Color bar: rock porosity of each sub-volume

\section{Model for computational experiments}

We assume that the studied rock volume consists of $5 \times 5 \times 5$ sub-volumes and $10 \times 10 \times 10$ sub-volumes respectively. Then we conduct computational experiments using the two rock partition schemes. Each sub-volume has independent porosity and clay content calculated by Monte-Carlo simulation with a Gaussian probability distribution function. The mean porosity and mean clay content of the rock volume are 0.25 and 0.30 respectively, and both deviations are 0.07 . The porosity and clay content distribution for $10 \times 10 \times 10$ sub-volumes is shown in Fig. 2. For each sub-volume, there are two minerals: clay and quartz, making up the rock framework and the pore fluid in the wet rock is water. Their properties used in computational experiments are shown in Table 1 (Batzle and Wang, 1992; Mavko et al, 2009).
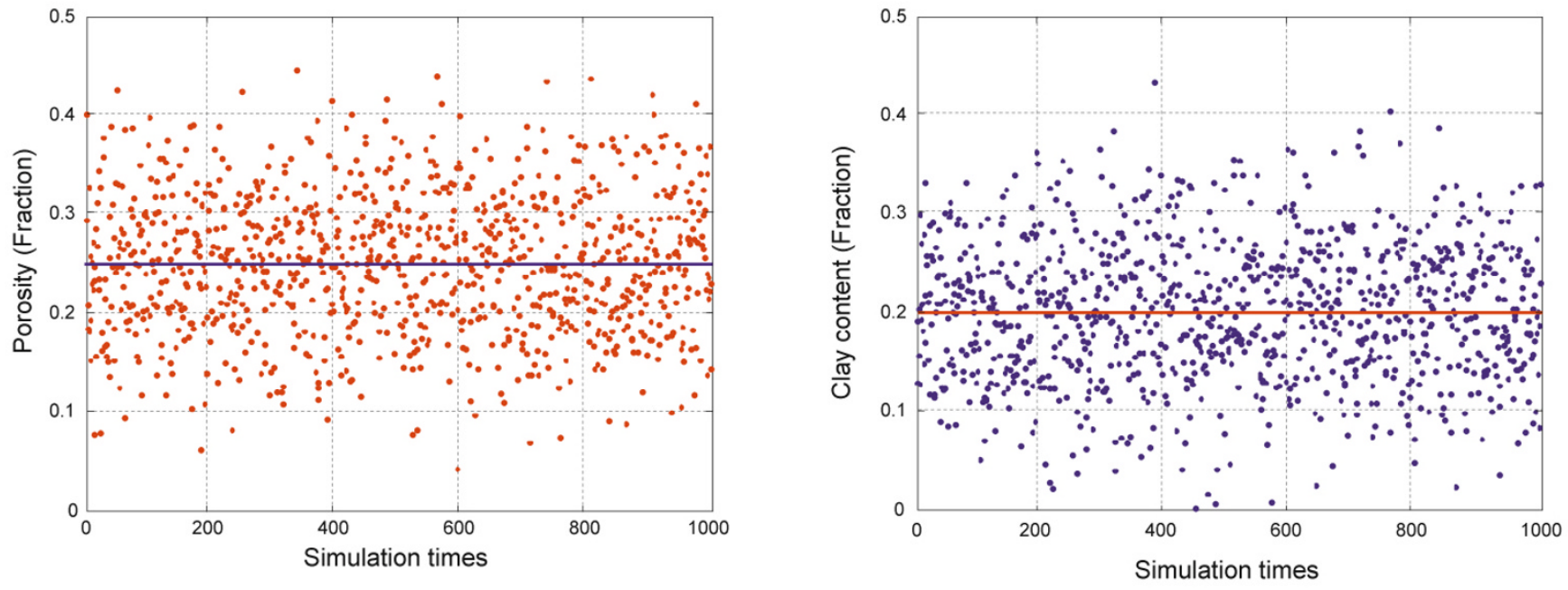

Fig. 2 Left: The porosity distribution, the line is the mean porosity of the rock volume. Right: The clay content distribution, the line is the mean clay content of the rock volume 
Table 1 Mineral and fluid properties used in computational experiments

\begin{tabular}{cccc}
\hline Component & Bulk moduli, GPa & Shear moduli, GPa & Density, g/cc \\
\hline Quartz & 36.60 & 45.00 & 2.65 \\
Clay & 21.00 & 7.00 & 2.58 \\
Water & 2.32 & 0.00 & 0.96 \\
\hline
\end{tabular}

\section{Computational experiments}

To study the difference of calculated effective bulk moduli of shaley sandstone estimated from mean porosity and directly from the sub-volumes, we use rock physical models, including Han's shaley sandstone model, the soft-sand model, stiff-sand model, and their combination, to conduct computational experiments with same input parameters, including porosity, clay content and effective pressure. Han's model is from rock physical experiments, the others are from rock physical theory derivation but all of them are confirmed by real data. The stiff-sand model and the soft-sand model can be used respectively for the upper and lower bounds of shaley sand elastic moduli. So we get plausible results from these representative models. Han's dry shaley sandstone

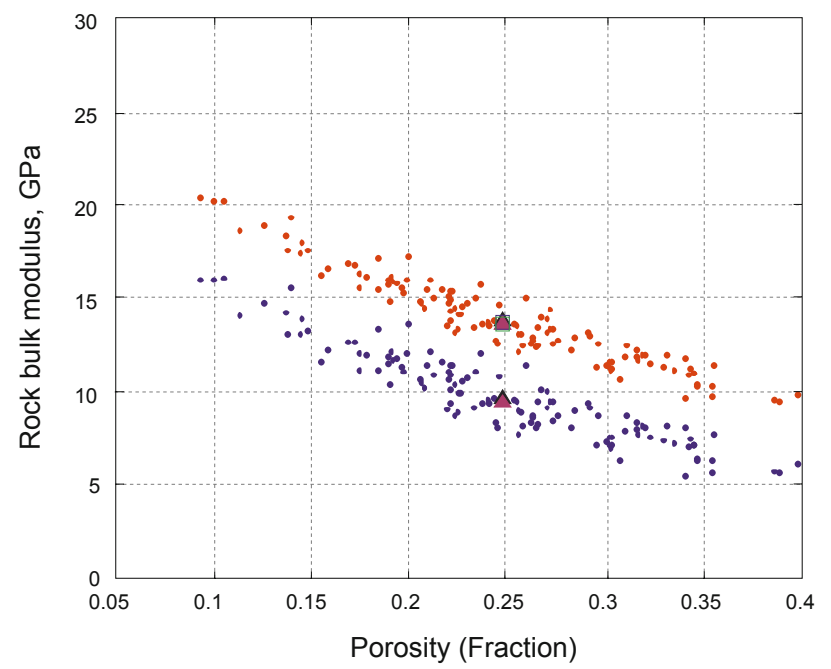

model under $40 \mathrm{MPa}$ effective pressure, shown as Eqs. (3) and (4), are derived from laboratory data measured on 70 shaley sandstone samples (Han et al, 1986). These laboratory data were measured using high-frequency waves but on dry samples. Because there is no fluid action in the rock, the frequency effect is negligible. We compute $V_{\mathrm{p}}$ and $V_{\mathrm{s}}$ of each dry sub-volume by Eqs. (3) and (4), and its density by volume averaging pore fluid, clay and quartz densities. We compute bulk and shear moduli of each dry sub-volume and the upper and lower bounds of the sub-volumes set by generalized Hashin-Shtrikman-Walpole bounds. We compute bulk moduli upper and lower bounds of the water-saturated rock from the bulk moduli upper and lower bounds of dry rock using mean porosity by Gassmann's equation. At the same time, we compute each sub-volume moduli of the water-saturated rock by Gassmann's equation from dry sub-volume moduli, and obtain the effective bulk moduli upper and lower bounds by generalized Hashin-Shtrikman-Walpole bounds. The computational data are shown as Fig. 3.

$$
\begin{aligned}
& V_{\mathrm{p}}=5.41-6.35 \varphi-2.87 C \\
& V_{\mathrm{s}}=3.57-4.57 \varphi-1.83 C
\end{aligned}
$$

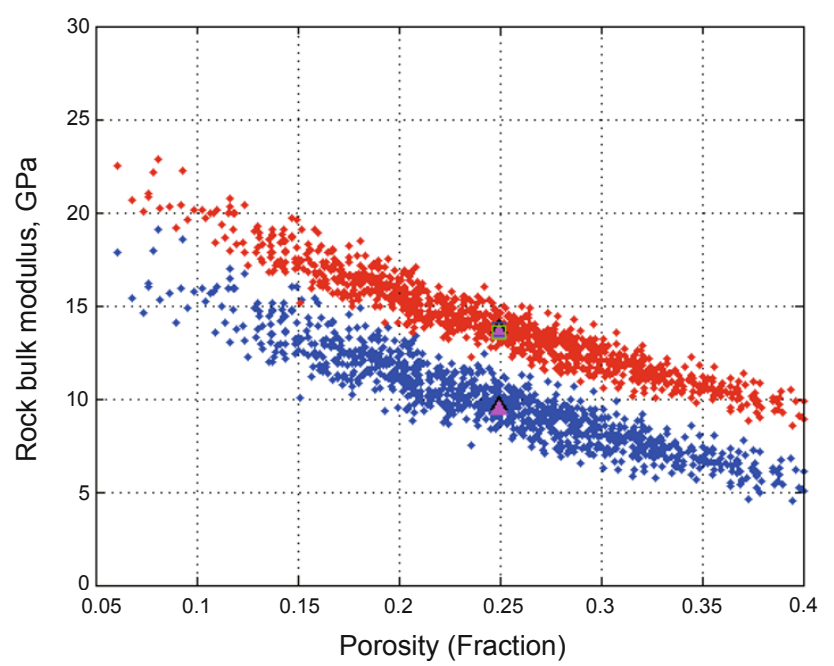

Fig. 3 Bulk modulus versus porosity by Han's shaley sand model. Left: the volume consists of $5 \times 5 \times 5$ sub-volumes; Right: the volume consists of $10 \times 10 \times 10$ sub-volumes. Red circles: the bulk moduli of dry rock versus porosity for each sub-volume. Blue circles: the bulk moduli of water-saturated rock versus porosity for each sub-volume. The black and pink triangles are bulk moduli upper and lower bounds by Hashin-Shtrikman-Walpole methods for dry and water-saturated rock in the first way described above. The blue and cyan squares are bulk moduli upper and lower bounds by HashinShtrikman-Walpole methods for water-saturated rock in the second way described above. (In the cases, the lower and upper bounds are very close to each other and appear as a single square.)

There is a big variation range for bulk moduli of dry-rock and water-saturated rock computed by Han's shaley sandstone model. But the differences are small between effective bulk moduli upper and lower bounds for both dry and watersaturated rock. The bulk moduli upper and lower bounds of water-saturated rock calculated by the second way described above overlap on those calculated by the first way. So at seismic scale, there is little difference in fluid substitution by the two ways. The rock partition schemes have little effect on the above conclusions.

Next, the soft-sand model is applied to calculate the bulk and shear moduli $K_{\text {eff }}, G_{\text {eff }}$ of dry sandstone in which cement is deposited away from grain contacts (Dvorkin and Nur, 1996). In this model a heuristic modified Hashin-Shtrikman lower bound (Hashin and Shtrikman, 1963) is used. The model is shown as Eqs. (5) and (6):

$$
K_{\text {eff }}=\left[\frac{\varphi / \varphi_{0}}{K_{\mathrm{HM}}+\frac{4}{3} G_{\mathrm{HM}}}+\frac{1-\varphi / \varphi_{0}}{K+\frac{4}{3} G_{\mathrm{HM}}}\right]^{-1}-\frac{4}{3} G_{\mathrm{HM}}
$$




$$
\begin{aligned}
G_{\mathrm{eff}} & =\left[\left[\frac{\varphi / \varphi_{0}}{G_{\mathrm{HM}}+\frac{G_{\mathrm{HM}}}{6}\left(\frac{9 K_{\mathrm{HM}}+8 G_{\mathrm{HM}}}{K_{\mathrm{HM}}+2 G_{\mathrm{HM}}}\right)}\right]+\left[\frac{1-\varphi / \varphi_{0}}{G+\frac{G_{\mathrm{HM}}}{6}\left(\frac{9 K_{\mathrm{HM}}+8 G_{\mathrm{HM}}}{K_{\mathrm{HM}}+2 G_{\mathrm{HM}}}\right)}\right]\right]^{-1} \\
& -\frac{G_{\mathrm{HM}}}{6}\left(\frac{9 K_{\mathrm{HM}}+8 G_{\mathrm{HM}}}{K_{\mathrm{HM}}+2 G_{\mathrm{HM}}}\right)
\end{aligned}
$$

In upper equations, $\varphi$ and $\varphi_{0}$ are the porosity and critical porosity, $K$ and $G$ are the bulk and shear moduli of rock grains, $K_{\mathrm{HM}}$ and $G_{\mathrm{HM}}$ are the effective bulk and shear moduli of randomly packed identical spherical grains under pressure calculated by contact Hertz-Mindlin theory (Mindlin, 1949; Hill, 1952). The soft-sand model is suited for dry unconsolidated shaley sand under following assumptions: (1) the strains are small; (2) grains are elastic, homogeneous;

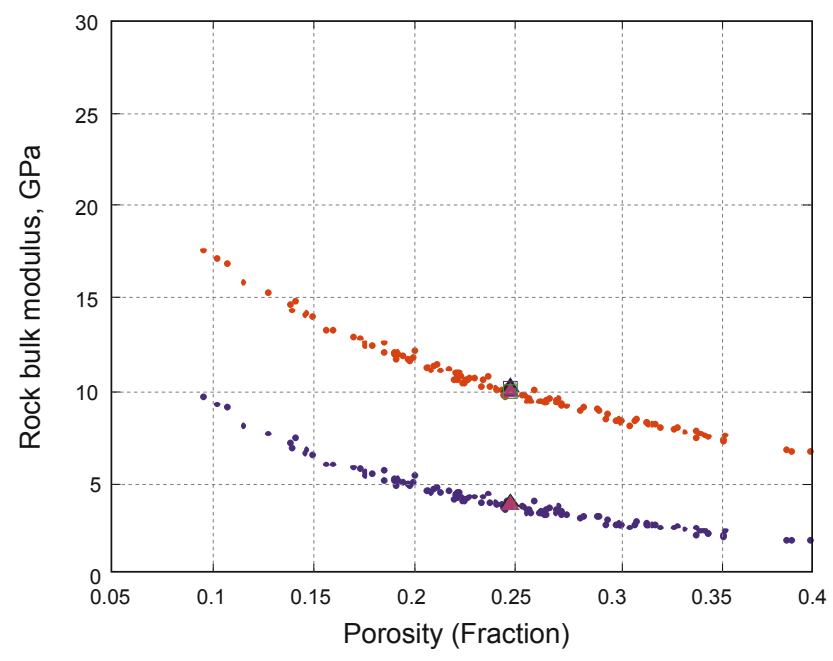

(3) packing is random and statistically isotropic; (4) the wavelength is much longer than the grain radius.

Using the same input parameters and ways as in Han's shaley sandstone model, we compute each sub-volume bulk modulus of dry and wet rock, and their upper and lower bounds of the sub-volumes set under $40 \mathrm{MPa}$ effective pressure by the two ways described above. The computational data are shown as Fig. 4.

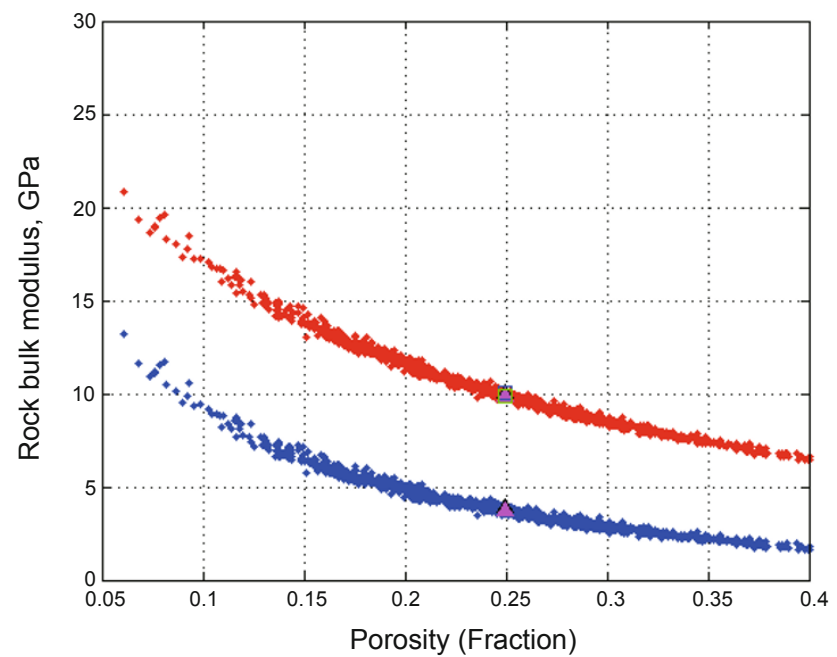

Fig. 4 Bulk moduli versus porosity by the soft-sand model. Left: the volume consists of $5 \times 5 \times 5$ sub-volumes; Right: the volume consists of $10 \times 10 \times 10$ subvolumes. Red circles: the bulk moduli of dry rock versus porosity for each sub-volume. Blue circles: the bulk moduli of water-saturated rock versus porosity for each sub-volume. The black and pink triangles are bulk moduli upper and lower bounds by Hashin-Shtrikman-Walpole methods for dry and water-saturated rock in the first way described above. The blue and cyan squares are bulk moduli upper and lower bounds by Hashin-Shtrikman-Walpole methods for watersaturated rock in the second way described above. (In the cases, the lower and upper bounds are very close to each other and appear as a single square.)

The fluid effect on bulk modulus computed by the softsand model is bigger than that by Han's shaley sand model. There is a smaller bulk modulus and variation range computed by the soft-sand model than those computed by Han's shaley sand model at a given porosity. The clay content has smaller effect on moduli under $40 \mathrm{MPa}$ effective pressure in the softsand model. The difference is smaller between effective bulk moduli upper and lower bounds of dry and water-saturated rock than that computed by Han's shaley sandstone model. The bulk moduli upper and lower bounds of water-saturated rock calculated by the second way also overlap on those calculated by the first way. So at seismic scale, the same conclusion is derived as obtained by Han's model.

The stiff-sand model for cemented sandstone is a counterpart to the soft-sand model (Mavko et al, 2009). The model uses precisely the same end-members as the soft-sand model, but connects them with a heuristic modified HashinShtrikman (Hashin and Shtrikman, 1963) upper bound. The stiff-sand model is suited for dry consolidated shaley sand under the same assumptions as the soft-sand model. The model is shown as Eqs. (7) and (8).

$$
\begin{aligned}
& K_{\mathrm{eff}}=\left[\frac{\varphi / \varphi_{0}}{K_{\mathrm{HM}}+\frac{4}{3} G}+\frac{1-\varphi / \varphi_{0}}{K+\frac{4}{3} G}\right]^{-1}-\frac{4}{3} G \\
& G_{\mathrm{eff}}=\left[\left[\frac{\varphi / \varphi_{0}}{G_{\mathrm{HM}}+\frac{G}{6}\left(\frac{9 K+8 G}{K+2 G}\right)}\right]+\left[\frac{1-\varphi / \varphi_{0}}{G+\frac{G}{6}\left(\frac{9 K+8 G}{K+2 G}\right)}\right]\right]^{-1} \\
& -\frac{G}{6}\left(\frac{9 K+8 G}{K+2 G}\right)
\end{aligned}
$$


In the upper equations, $\varphi$ and $\varphi_{0}$ are the porosity and critical porosity, $K$ and $G$ are the bulk and shear moduli of rock grains, $K_{\mathrm{HM}}$ and $G_{\mathrm{HM}}$ are the effective bulk and shear moduli of randomly packed identical spherical grains under pressure calculated by contact Hertz-Mindlin theory (Mindlin, 1949). Using the same input parameters and

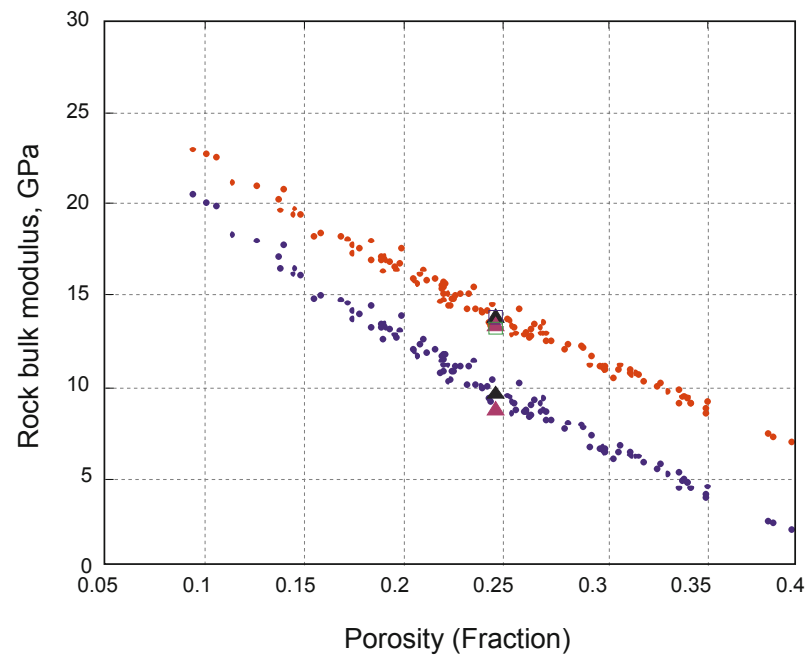

ways as in Han's shaley sandstone model and the soft-sand model study, we compute the bulk moduli of each subvolume of dry and water-saturated rock, and bulk moduli upper and lower bounds of the sub-volumes set under 40 MPa effective pressure. The computational data are shown as Fig. 5.

Fig. 5 Bulk moduli versus porosity by the stiff-sand model. Left: the volume consists of $5 \times 5 \times 5$ sub-volumes; Right: the volume consists of $10 \times 10 \times 10$ sub-volumes. Red circles: the bulk moduli of dry rock versus porosity for each sub-volume. Blue circles: the bulk moduli of watersaturated rock versus porosity for each sub-volume. The black and pink triangles are bulk moduli upper and lower bounds by Hashin-ShtrikmanWalpole methods for dry and water-saturated rock in the first way described above. The blue and cyan squares are bulk moduli upper and lower bounds by Hashin-Shtrikman-Walpole methods for water-saturated rock in the second way described above

The fluid effect on rock bulk moduli computed by the stiff-sand model is smaller than that by the soft-sand model, because there are bigger rock bulk moduli in the stiff-sand model. In a given porosity, there are bigger bulk modulus and variation range computed by the stiff-sand model than those computed by the soft-sand model. The clay content has a bigger effect on bulk moduli under $40 \mathrm{MPa}$ effective pressure in the stiff-sand model than in the soft-sand model. So there is a bigger difference between effective bulk moduli upper and lower bounds for dry and water-saturated rock than that computed by the soft-sand model. The bulk moduli upper and lower bounds of water-saturated rock calculated by the second way also overlap on those calculated by the first way. So at seismic scale, the same conclusion can be drawn as obtained by Han's model and the soft-sand model.

Next, we conduct computational experiments on the combination of the soft-sand model and the stiff-sand model, that is, the rock is made up of soft sand and stiff sand half and half. With the same input parameters and by the same ways as above, we get the computational data shown as Fig. 6 . The bulk moduli of dry and water-saturated rock are both separated because there are big differences of elastic properties for the two kinds of sands. The bulk moduli upper and lower bounds of water-saturated rock computed by the second way almost overlap on those by the first way, but the difference is bigger than that in the above model for stronger rock heterogeneity.

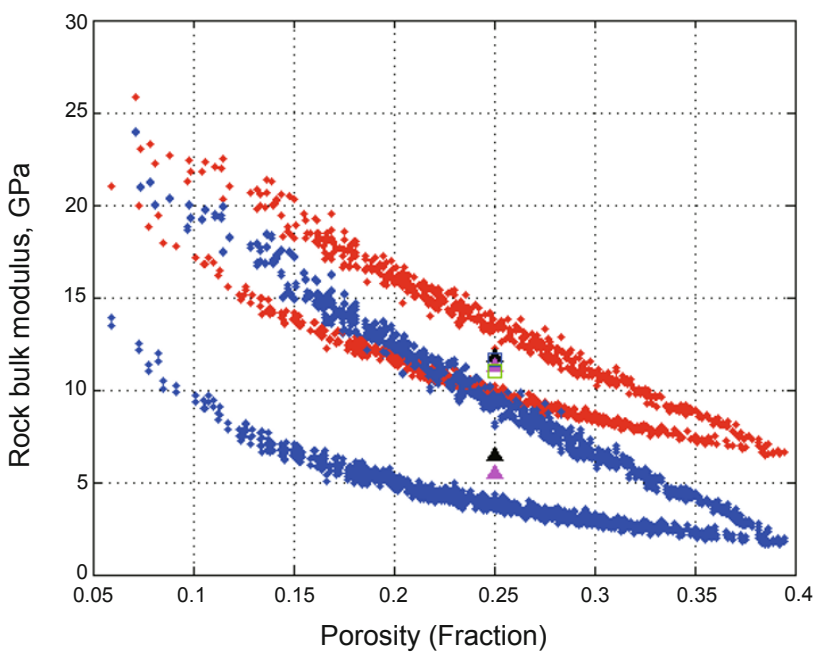

Fig. 6 Rock bulk moduli versus porosity by combined model of the stiffsand model and the soft-sand model. Red circles: the bulk moduli of dry rock versus porosity for each sub-volume. Blue circles: the bulk moduli of water-saturated rock versus porosity for each sub-volume. The black and pink triangles are bulk moduli upper and lower bounds by HashinShtrikman-Walpole methods for dry and water-saturated rock in the first way described above. The blue and cyan squares are bulk moduli upper and lower bounds by Hashin-Shtrikman-Walpole methods for watersaturated rock in the second way described above

\section{Analysis and discussion}

In Han's shaley sand model, the soft-sand model and the 
stiff-sand model, the variations of porosity and clay content affect the rock bulk modulus to different degrees, shown as Fig. 7. But for all the three models, the differences are small between the upper and lower bounds of dry and/or

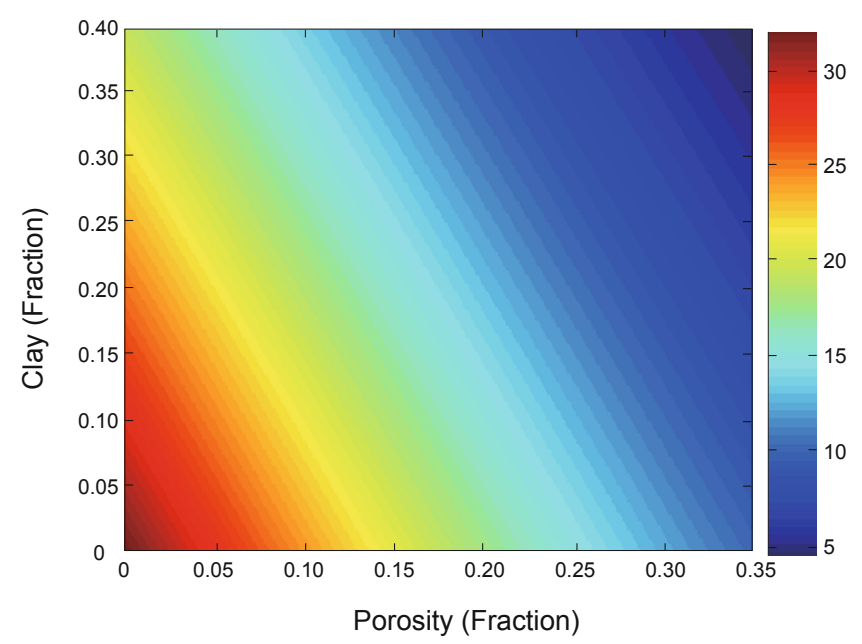

(a)

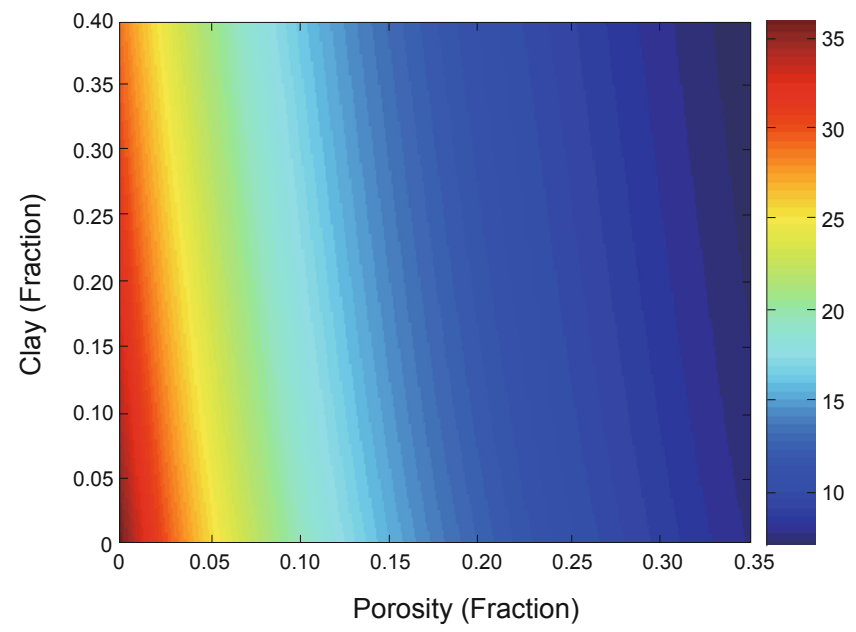

(b)

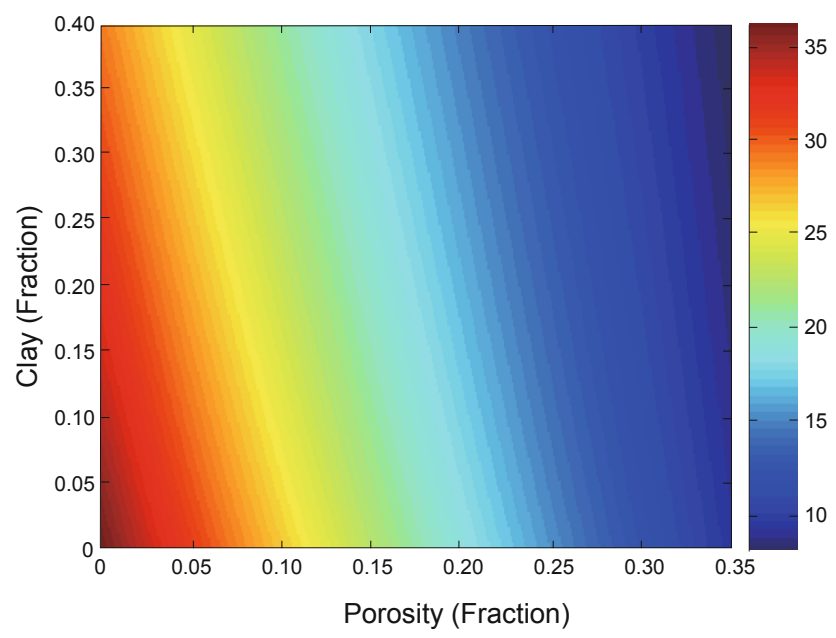

(c)

Fig. 7 The color-coded bulk modulus versus porosity and clay content according to (a) Han's model, (b) the soft-sand model, and (c) the stiff-sand model. Color bar: bulk modulus, unit: GPa wet rock bulk moduli. Since there are small differences, we can represent the rock effective moduli with their average $\left(\left(M^{\mathrm{HS}+}+M^{\mathrm{HS}-}\right) / 2\right)$. We compute the effective bulk moduli of dry and water-saturated sands with varying average porosities using the stiff-sand model and the same two ways described above. In the computational experiments, fluid substitutions are conducted in all the rock sub-volumes. The computational results (Fig. 8: Left) show that there are very small differences between effective moduli calculated by mean porosity and those calculated directly from the subvolumes. But for shaley sandstone under seismic scale, the porosity and clay content have big variation ranges. Fluid substitutions are impossible under real reservoir conditions for the sub-volumes with too small porosity and/or too high clay content. So there are differences between effective bulk moduli by mean porosity and directly from the sub-volumes in real reservoir conditions (Fig. 8: Right). And there are more sub-volumes without fluid substitution in the rock
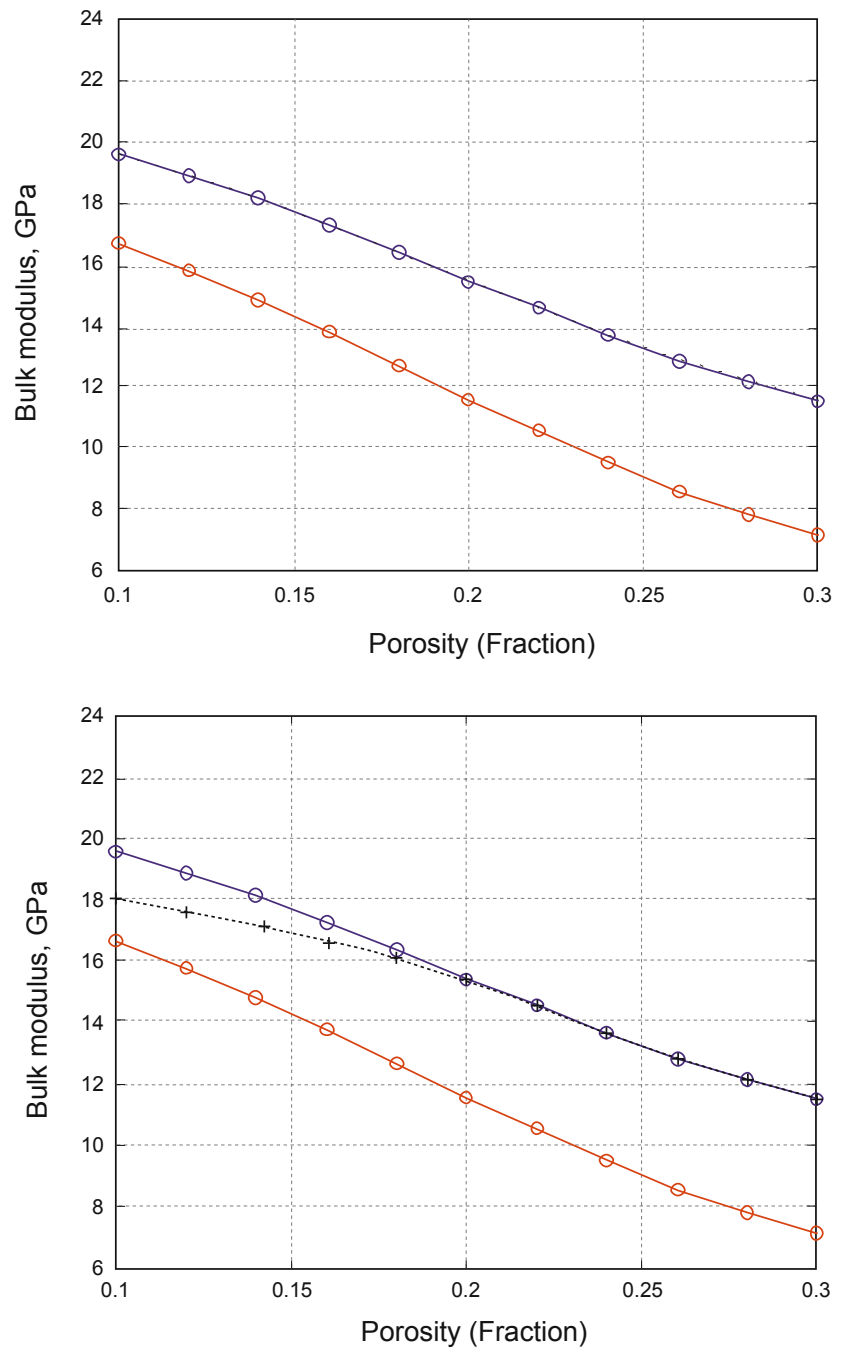

Fig. 8 Average bulk moduli versus average porosity by the stiff-sand model. Upper: fluid substitution in all sub-volumes; Lower: no fluid substitution in the sub-volumes with low porosity (under 5\%) and high clay content (higher than $40 \%$ ). Red line with circle: the dry rock average bulk moduli versus porosity. Blue line with circle: the average bulk moduli of water-saturated rock versus porosity calculated by mean porosity. Black dash line with cross: the average bulk moduli of watersaturated rock versus porosity calculated directly from the sub-volumes 
with smaller mean porosity. In all probability, this is one of important reasons for Gassmann's equation does not work as well in low porosity and high clay content rock as in high porosity rock.

We also compute the average bulk moduli (as explained above) of dry and water-saturated shaley sands with varying average porosities using the combination of stiff-sand model and soft-sand model and the same two ways described in the above text. In the computational experiments, fluid substitutions are conducted in all the rock sub-volumes. The computational results (Fig. 9) show that there are visible bulk moduli differences by the two ways because elastic property differences between the soft sand model and the stiff-sand model produce a rock with stronger heterogeneity.

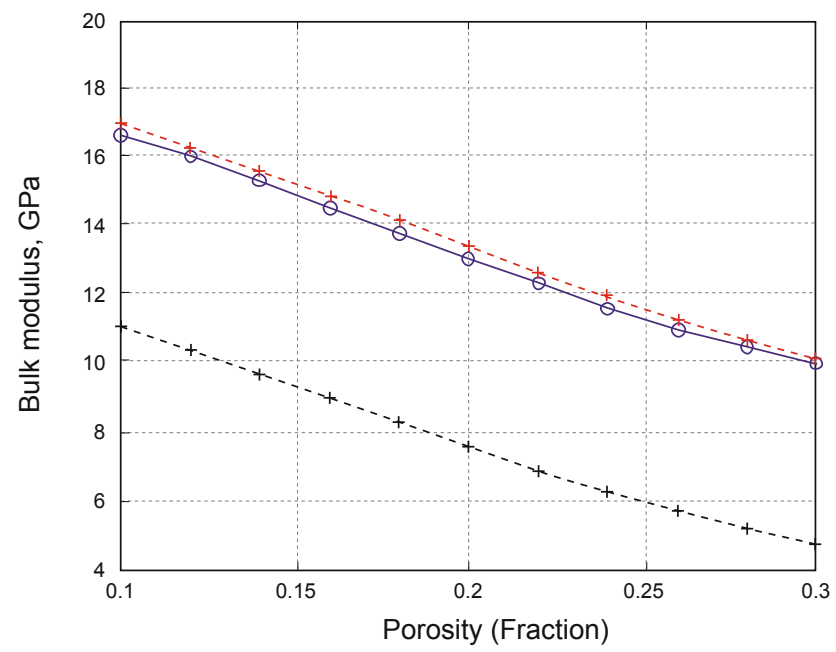

Fig. 9 Average bulk modulus versus average porosity by the stiff-sand model. Black dash line with cross: the average bulk moduli of dry rock versus average porosity. Blue line with circle: the average bulk moduli of water-saturated rock versus average porosity calculated by mean porosity. Red dash line with cross: the average bulk moduli of watersaturated rock versus porosity calculated directly from the sub-volumes

\section{Conclusions and suggestion}

The computational experiments on shaley sand rock physical models show that there are minor fluid substitution differences between bulk moduli calculated by mean porosity and those calculated directly from the sub-volumes using Gassmann's equation. The differences are related to the bulk moduli variation range in rock volume. But under real reservoir conditions, too small a porosity and/or too high a clay content in some sub-volumes make fluid substitution impossible, so fluid substitution by mean porosity using Gassmann's equation likely gives a bigger variation than that in real reservoir conditions, especially for low porosity and/or high clay content rock.

\section{Acknowledgements}

This study is financially supported by National Natural Science Function of China (No. 41074098) and National 973 Basic Research Program (No. 2007CB209606). I thank senior scientist Dr. Jack Dvorkin in Stanford University for his valuable instruction and suggestions.

\section{References}

Avseth P, Mukerji T and Mavko G. Quantitative Seismic Interpretation. New York: Cambridge University Press. 2005. 136-137

Batzle $M$ and Wang Z. Seismic properties of pore fluids. Geophysics. 1992. 57(11): 1396-1408

Berryman J G. Mixture theories for rock properties. In: Rock Physics and Phase Relations: a Handbook of Physical Constants, ed. by Ahrens T J. Washington, DC: American Geophysical Union. 1995. 205-228

Blangy J D. Integrated seismic lithologic interpretation: The petrophysical basis. Stanford University Ph.D. Thesis. 1992. 238-290

Dvorkin J and Nur A. Elasticity of high-porosity sandstones: Theory for two North Sea data sets. Geophysics. 1996. 61(5): 1363-1370

Dvorkin J and Uden R. The challenge of scale in seismic mapping of hydrate and solutions. The Leading Edge. 2006. 25(5): 637-642

Dvorkin J, Mavko G and Gurevich B. Fluid substitution in shaley sediment using effective porosity. Geophysics. 2007. 72(3): 1-8

Gassmann F. Elasticity of porous media: Uber die elastizitat poroser medien: Vierteljahrsschrift der Naturforschenden. Gesselschaft. 1951. 96(1): 1-23

Han D H. Effects of porosity and clay content on acoustic properties of sandstones and unconsolidated sediments. Stanford University Ph.D. Thesis. 1987. 53-94

Han D H, Nur A and Morgan D. Effects of porosity and clay content on wave velocities in sandstones. Geophysics. 1986. 51(4): 2093-2107

Hashin Z and Shtrikman S. A variational approach to the theory of the elastic behavior of multiphase materials. Journal of the Mechanics and Physics of Solids. 1963. 11(2): 127-140

Hill R. The elastic behavior of a crystalline aggregate. Proceedings of the Physical Society, Section A. 1952. 65(5): 349-354

Mavko G, Mukerji T and Dvorkin J. The Rock Physics Handbook. New York: Cambridge University Press. 2009. 245-265

Mindlin R D. Compliance of elastic bodies in contact. Journal of Applied Mechanics. 1949. 16(3): 259-268

Skelt C. Fluid substitution in laminated sands. The Leading Edge. 2004. 23(5): 485-493

(Edited by Hao Jie) 